\title{
Sparse tensor edge elements
}

\section{Journal Article}

\section{Author(s):}

Hiptmair, Ralf (D); Jerez-Hanckes, Carlos; Schwab, Christoph

Publication date:

2013-12

\section{Permanent link:}

https://doi.org/10.3929/ethz-b-000075125

Rights / license:

In Copyright - Non-Commercial Use Permitted

Originally published in:

BIT Numerical Mathematics 53(4), https://doi.org/10.1007/s10543-013-0435-3 


\title{
Sparse tensor edge elements
}

\author{
Ralf Hiptmair • Carlos Jerez-Hanckes • \\ Christoph Schwab
}

Received: 4 November 2012 / Accepted: 23 April 2013 / Published online: 27 June 2013

(C) Springer Science+Business Media Dordrecht 2013

\begin{abstract}
We consider the tensorized operator for the Maxwell cavity source problem in frequency domain. Such formulations occur when computing statistical moments of the fields under a stochastic volume excitation. We establish a discrete inf-sup condition for its Ritz-Galerkin discretization on sparse tensor product edge element spaces built on nested sequences of meshes. Our main tool is a generalization of the edge element Fortin projector to a tensor product setting. The techniques extend to the surface boundary edge element discretization of tensorized electric field integral equation operators.
\end{abstract}

Keywords Sparse tensor approximation - Stochastic source problems · Maxwell cavity source problem $\cdot$ Edge elements $\cdot$ Fortin projector $\cdot$ Commuting diagram property

Mathematics Subject Classification (2010) 78M15 - 65C30 - 65N38 - 60H35

Communicated by Ragnar Winther.

The work of C. Schwab was partially supported by the European Research Council under grant number ERC AdG 247277-STAHDPDE.

C. Jerez-Hanckes's work was partially funded by FONDECYT 11121166 and CONICYT project Anillo ACT1118 (ANANUM).

R. Hiptmair $(\varangle) \cdot$ C. Schwab

SAM, ETH Zürich, 8092 Zürich, Switzerland

e-mail: hiptmair@sam.math.ethz.ch

C. Schwab

e-mail: schwab@sam.math.ethz.ch

C. Jerez-Hanckes

School of Engineering, Pontificia Universidad Católica de Chile, Santiago, Chile

e-mail: cjerez@ing.puc.cl 


\section{Second moment problem}

Let $V$ be a Hilbert space and consider an isomorphism $\mathrm{A}: V \rightarrow V^{\prime}$. If the right hand side of the operator equation $\mathrm{A} u=f$ is "stochastic" in the sense that it belongs to $L^{2}\left(\Omega, V^{\prime}\right)$ for a probability space $(\Omega, \mathcal{A}, \mathbb{P})$, then also the solution $u$ becomes a $V$-valued square integrable random variable: $u \in L^{2}(\Omega, V)$. Its second moment $\mathcal{M}^{2} u=\mathbb{E}(u \otimes u) \in L^{1}(V \otimes V)$, where $\mathbb{E}$ denotes the expectation, can be obtained as the solution of

$$
(\mathbf{A} \otimes \mathbf{A}) w^{(2)}=\mathcal{M}^{2} f
$$

featuring the tensor product operator $\mathrm{A} \otimes \mathrm{A}: V \otimes V \rightarrow V^{\prime} \otimes V^{\prime}$. Well-known results guarantee existence and uniqueness of solutions of this equation; see [15, Sect. 1] for a comprehensive exposition.

A stable Ritz-Galerkin discretization of $\mathrm{A} u=f$ by means of a finite dimensional trial space $V_{h} \subset V$ immediately spawns a stable Ritz-Galerkin discretization of (1.1), when using the "full tensor product" trial and test space $V_{h}^{(2)}:=V_{h} \otimes V_{h}$. Unfortunately, $\operatorname{dim} V_{h}^{(2)}=\left(\operatorname{dim} V_{h}\right)^{2}$, whereas the approximation power of $\operatorname{dim} V_{h}^{(2)}$ is usually not better than that of $V_{h}$. This is the notorious "curse of dimensionality".

Taking for granted smoothness of $\mathcal{M}^{2} u$, a remedy is offered by sparse tensor Galerkin discretization, using subspaces $\widehat{V}_{h}^{(2)}$ of $V_{h}^{(2)}$ with approximation power almost like that of $V_{h}$, but dimensions substantially reduced to $\operatorname{dim} \widehat{V}_{h}^{(2)} \approx \operatorname{dim} V_{h}$, see [15, Sect. 1.4].

However, the stability of sparse tensor Galerkin discretizations can no longer be inferred from that for $V_{h}$ applied to A, unless $\mathrm{A}$ is positive. Non-positive operators are invariably encountered in wave propagation phenomena in frequency domain, and for them stability of the sparse tensor Galerkin discretization has to be established directly. This was done for boundary value problems for the Helmholtz equation $-\Delta u-k^{2} u=f$ in [16], see also [15, Sect. 1.4]. In the present article we are going to tackle the issue for the Maxwell cavity source problem in frequency domain and its discretization by means of edge elements.

Upon finishing the first version of this article, the authors learned about the recent report [4], which studies tensor product operators arising from mixed variational problems and their sparse tensor discretization based on discrete differential forms. The techniques employed in this parallel work are similar to ours and, in particular, the steps (1- $\mathbf{7}$ of the proof of Theorem 5.1 given in Sect. 5.2, are also followed in the proof of [4, Lemma E.1, Erratum].

\section{Maxwell cavity operator}

From now on, $V:=\boldsymbol{H}_{0}\left(\right.$ curl, $D$ ) for a Lipschitz polyhedron $D \subset \mathbb{R}^{3}$ and the operator A : $V \rightarrow V^{\prime}$ is induced by the continuous sesquilinear form

$$
a(\mathbf{u}, \mathbf{v}):=(\operatorname{curl} \mathbf{u}, \operatorname{curl} \mathbf{v})_{L^{2}(D)}-k^{2}(\mathbf{u}, \mathbf{v})_{L^{2}(D)}, \quad \mathbf{u}, \mathbf{v} \in V,
$$


where the wave number $k>0$ is supposed to be different from a resonant frequency of $D, c f$. [10, Ass. 1]. This guarantees that $\mathrm{A}$ is bijective, that is, it satisfies an infsup-condition. As explained in [10, Sect. 5.1] the proof of this fact can make use of the $V$-orthogonal Helmholtz decomposition: ${ }^{1}$

$$
\begin{array}{ll}
V=X \oplus Z, \quad Z:=\boldsymbol{H}_{0}(\operatorname{curl} 0, D):=V \cap \operatorname{kern}(\operatorname{curl}), \\
X \subset \boldsymbol{H}(\operatorname{div} 0, D) \cap \boldsymbol{H}_{0}(\operatorname{curl}, D) .
\end{array}
$$

Its components are closed subspaces of $V$ [10, Lemma 2.2], and functions in $X$ possess extra regularity, which renders the embedding $X \hookrightarrow\left(L^{2}(D)\right)^{3}$ compact [10, Thm 4.1]. The Helmholtz decomposition induces two $V$-orthogonal projectors $\mathrm{P}_{X}: V \rightarrow X$ and $\mathrm{P}_{Z}: V \rightarrow Z$, which enter the definition of the sign-flipping isomorphism, cf. [5, Ass. 1], [6, Sect. 4], [8, Sect. 4.4],

$$
\Theta:=\mathrm{P}_{X}-\mathrm{P}_{Z}=2 \mathrm{P}_{X}-\mathrm{Id}: V \rightarrow V .
$$

It is a key ingredient of the following generalized Gårding inequality, that asserts the existence of a compact operator $\mathrm{K}: V \rightarrow V^{\prime}$ such that

$$
\left|a(\mathbf{u}, \boldsymbol{\Theta} \mathbf{u})+\langle\mathrm{Ku}, \overline{\mathbf{u}}\rangle_{V^{\prime} \times V}\right| \geq C_{\text {stab }}\|\mathbf{u}\|_{V}^{2} \quad \forall \mathbf{u} \in V,
$$

with $C_{\text {stab }}>0$ depending only on $k$ and $D$, see [10, (5.8)] and [5, (1.1)].

\section{Edge element spaces}

We start from a shape-regular sequence of nested tetrahedral triangulations of $D$ : $\mathcal{T}_{0} \prec \mathcal{T}_{1} \prec \ldots \prec \mathcal{T}_{l} \prec \ldots$, for instance, created by successive global regular refinement of $\mathcal{T}_{0}$. Thus, the index $l$ should be read as a "level of refinement". The sequence of mesh-widths $\left(h_{l}\right)_{l}$ of $\left(\mathcal{T}_{l}\right)_{l}$ is supposed to decrease geometrically:

$$
h_{l} \leq h_{0} q^{l} \text { for some } 0<q<1 .
$$

We write $\mathcal{W}_{h}^{1}\left(\mathcal{T}_{l}\right)$ for the finite-dimensional space of lowest order edge elements on $\mathcal{T}_{l}$ [10, Sect. 3.2] (also known as Whitney-1-forms or lowest order Nédélec elements of the first family [12]) and will often use the abbreviation $V_{l}:=\mathcal{W}_{h}^{1}\left(\mathcal{T}_{l}\right)$. We point out that these spaces are nested in the sense that $V_{l-1} \subset V_{l}$ and that they are asymptotically dense in $V$. Thus, the sequence $\left(\mathrm{P}_{l}\right)_{l}$ of $V$-orthogonal projectors $\mathrm{P}_{l}: V \rightarrow V_{l}$ converges to Id pointwise, $c f$. [10, Lemma 5.5].

The spaces $V_{l}, l \in \mathbb{N}_{0}$, provide an asymptotically stable Ritz-Galerkin discretization of the bilinear form $a$ from (2.1) [10, Thm. 5.7]. As highlighted in [10, Sect. 5.2], commuting projectors are instrumental for the proof. They will also play a pivotal role in our considerations; we rely on particular specimens, called Fortin projectors [10, Sect. 4.2] introduced by D. Boffi in [3].

\footnotetext{
${ }^{1}$ We adopt the customary notations for function spaces also used in [10], for instance, $\boldsymbol{H}(\operatorname{div} 0, D):=\{\mathbf{v} \in$ $\boldsymbol{H}(\operatorname{div}, D): \operatorname{div} \mathbf{v}=0\}$, and a zero subscript indicates vanishing trace on $\partial \Omega$.
} 
To define them, let us write $\mathcal{W}_{h}^{2,0}\left(\mathcal{T}_{l}\right):=\operatorname{curl} \mathcal{W}_{h}^{1}\left(\mathcal{T}_{l}\right) \subset \boldsymbol{H}_{0}(\operatorname{div} 0, D)$ and, slightly abusing notation, $\mathcal{W}_{h}^{1,0}\left(\mathcal{T}_{l}\right):=\mathcal{W}_{h}^{1}\left(\mathcal{T}_{l}\right) \cap \boldsymbol{H}(\operatorname{curl} 0, D)$ for spaces of irrotational finite element functions. The $L^{2}(D)$-orthogonal projections onto these spaces are denoted by $\mathrm{Q}_{l}:\left(L^{2}(D)\right)^{3} \rightarrow \mathcal{W}_{h}^{2,0}\left(\mathcal{T}_{l}\right)$ and $\widetilde{\mathrm{Q}}_{l}:\left(L^{2}(D)\right)^{3} \rightarrow \mathcal{W}_{h}^{1,0}\left(\mathcal{T}_{l}\right)$. The fact that the $L^{2}(D)$-orthogonal discrete Helmholtz decompositions

$$
\mathcal{W}_{h}^{1}\left(\mathcal{T}_{l}\right)=X_{l} \oplus \mathcal{W}_{h}^{1,0}\left(\mathcal{T}_{l}\right),
$$

are $l$-uniformly $V$-stable [10, Thm. 4.7], guarantees the existence of $l$-uniformly bounded surjective lifting operators

$$
\mathrm{L}_{l}: \mathcal{W}_{h}^{2,0}\left(\mathcal{T}_{l}\right) \rightarrow X_{l} \quad \text { such that } \quad \text { curl o } \mathrm{L}_{l}=\mathrm{ld} .
$$

Then, we define Fortin projectors ${ }^{2} \mathrm{~F}_{l}: V \rightarrow V_{l}$ as

$$
\mathrm{F}_{l}:=\mathrm{L}_{l} \circ \mathrm{Q}_{l} \circ \operatorname{curl}+\widetilde{\mathrm{Q}}_{l}
$$

This definition of the Fortin projector agrees with the abstract commuting discrete co-chain projector constructed in the proof of Thm. 3.7 of [1]. However, the approximation properties of these operators in $L^{2}$ are not discussed in [1]. The projectors inherit uniform stability from the liftings: ${ }^{3}$

$$
\left\|\mathrm{F}_{l} \mathbf{u}\right\|_{V} \leq C\|\mathbf{u}\|_{V} \quad \forall \mathbf{u} \in V, \quad \forall l \in \mathbb{N}_{0},
$$

and fulfill the obvious commuting diagram property

$$
\text { curl } \circ \mathrm{F}_{l}=\mathrm{Q}_{l} \circ \text { curl on } V \text {. }
$$

Since curl $\circ \widetilde{\mathrm{Q}}_{l}=0$ and $\widetilde{\mathrm{Q}}_{l} \circ \mathrm{L}_{l}=0, \mathrm{~F}_{l}$ is a surjective projector:

$$
\mathrm{F}_{l} \circ \mathrm{F}_{l}=\mathrm{F}_{l} \quad \text { and } \quad \mathrm{F}_{l}\left(\mathbf{v}_{l}\right)=\mathbf{v}_{l} \quad \forall \mathbf{v}_{l} \in \mathcal{W}_{h}^{1}\left(\mathcal{T}_{l}\right) .
$$

A deeper result about Fortin projectors is their approximation property in $X$ :

Lemma 3.1 There is $C>0$ and some $0<\epsilon \leq 1$ such that

$$
\left\|\left(\mathrm{ld}-\mathrm{F}_{l}\right) \mathbf{u}^{\perp}\right\|_{L^{2}(D)} \leq C h_{l}^{\epsilon}\left\|\mathbf{u}^{\perp}\right\|_{V} \quad \forall \mathbf{u}^{\perp} \in X, \quad \forall l \in \mathbb{N}_{0} .
$$

Proof We point out that $\widetilde{\mathrm{Q}}_{l}(X)=\{0\}$ and $\mathrm{L}_{l} \circ \mathrm{Q}_{l} \circ$ curl agrees with the operator $\mathrm{F}_{h}$ introduced in $[10,(4.10)]$. Then we can appeal to $[10, \mathrm{Thm} .4 .8]$ or the approximation results from [3].

Fortin projectors on different levels commute:

\footnotetext{
${ }^{2}$ Our Fortin projector agrees with the operator $\widetilde{F}_{h}$ defined on p. 311 of [10], but not the operator $\mathrm{F}_{h}$ defined on p. 297 of that survey.

${ }^{3}$ As usual, generic constants will be denoted by $C$. They may depend only on $D$ or the shape-regularity of the triangulations. Specific constants may be tagged with a subscript.
} 


\section{Lemma 3.2}

$$
\mathrm{F}_{l-1} \circ \mathrm{F}_{l}=\mathrm{F}_{l-1}=\mathrm{F}_{l} \circ \mathrm{F}_{l-1} \quad \text { for all } l \in \mathbb{N}_{0} \text {. }
$$

Proof Nested meshes lead to nested spaces $\mathcal{W}_{h}^{2,0}\left(\mathcal{T}_{l-1}\right) \subset \mathcal{W}_{h}^{2,0}\left(\mathcal{T}_{l}\right)$ and $\mathcal{W}_{h}^{1,0}\left(\mathcal{T}_{l-1}\right)$ $\subset \mathcal{W}_{h}^{1,0}\left(\mathcal{T}_{l}\right)$, with the simple consequence that for the $L^{2}$-projections

$$
\mathrm{Q}_{l-1} \circ \mathrm{Q}_{l}=\mathrm{Q}_{l-1}, \quad \widetilde{\mathrm{Q}}_{l-1} \circ \widetilde{\mathrm{Q}}_{l}=\widetilde{\mathrm{Q}}_{l-1}, \quad \widetilde{\mathrm{Q}}_{l-1} \circ \mathrm{L}_{l}=0 .
$$

From curl $\circ \widetilde{Q}_{l}=0$ and (3.3) we conclude the assertion.

Remark 3.1 In [4] the authors rely on so-called commuting co-chain projectors introduced and explored in $[1,9,14]$. Those could also substitute the Fortin projectors in our theory.

\section{Sparse tensor space}

As regards to the Ritz-Galerkin discretization of (1.1) with A from (2.1), a more economical finite dimensional subspaces of the full tensor edge element spaces $V_{L} \otimes$ $V_{L} \subset V \otimes V, V_{L}:=\mathcal{W}_{h}^{1}\left(\mathcal{T}_{L}\right), V=\boldsymbol{H}_{0}(\mathbf{c u r l}, D)$, are the sparse tensor edge element spaces, $c f$. [16, Def. 5.1], [15, Def. 1.17], [4, Sect. 6.4],

$$
\begin{gathered}
\widehat{V}_{L, L_{0}}:=\sum_{(l, k) \in \mathcal{S}_{L, L_{0}}} V_{l} \otimes V_{k}, \\
\mathcal{S}_{L, L_{0}}:=\left\{(l, k) \in\{0, \ldots, L\}^{2}, l+k \leq L+L_{0}\right\}, \quad 0 \leq L_{0} \leq L,
\end{gathered}
$$

of resolution $L$ and base level $L_{0}$, see Fig. 1 .

Remark 4.1 The base level $L_{0}$ ensures a minimal resolution "in both directions" in the sense that $V_{L_{0}} \otimes V_{L}, V_{L} \otimes V_{L_{0}} \subset \widehat{V}_{L, L_{0}}$. As discovered in [16, Sect. 5], thus we can accommodate the minimal resolution requirement, which is typical of the stable Ritz-Galerkin discretization of coercive, but non-positive variational problems [13]. Below in Sect. 5 the possibility to adjust $L_{0}$ will be crucial.

The sparse tensor space also allows a direct sum representation by means of the "surplus spaces":

$$
W_{l}:=\left(\mathrm{F}_{l}-\mathrm{F}_{l-1}\right)\left(V_{l}\right), \quad l \geq 1, \quad W_{0}:=V_{0},
$$

where $F_{l}$ are the Fortin projectors introduced in (3.4). Thanks to Lemma $3.2 V_{L}=$ $W_{0}+\cdots+W_{L}$ is a direct splitting, which implies that

$$
\widehat{V}_{L, L_{0}}=\sum_{(l, k) \in \mathcal{S}_{L, L_{0}}} W_{l} \otimes W_{k},
$$

is direct, as well. 


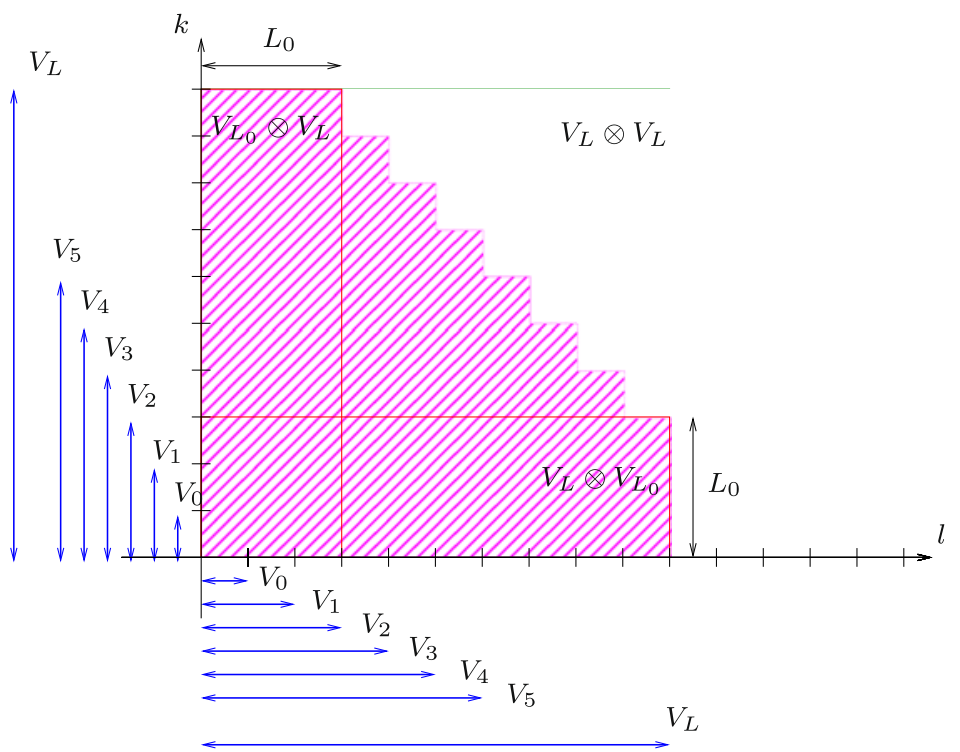

Fig. 1 Visualization of sparse tensor space $\widehat{V}_{L, L_{0}}$ (hatched area)

The linchpin of our approach is a "Fortin-type projector" onto the sparse tensor product space. Taking the cue from (4.2) and (4.3) we define

$$
\widehat{\mathrm{F}}_{L, L_{0}}^{(2)}:=\sum_{(l, k) \in \mathcal{S}_{L, L_{0}}} \Delta \mathrm{F}_{l} \otimes \Delta \mathrm{F}_{k}, \quad \Delta \mathrm{F}_{l}:=\mathrm{F}_{l}-\mathrm{F}_{l-1}, \quad \Delta \mathrm{F}_{0}:=\mathrm{F}_{0} .
$$

Lemma 4.1 The linear operator $\widehat{\mathrm{F}}_{L, L_{0}}^{(2)}: V \otimes V \rightarrow \widehat{V}_{L, L_{0}}$ is a bounded and surjective projector.

Proof Boundedness of $\widehat{F}_{L, L_{0}}^{(2)}$ follows from (3.5). Owing to Lemma 3.2 we find $\Delta \mathrm{F}_{l} \circ \Delta \mathrm{F}_{m}=0$ for $l \neq m$, from which we infer the projector property. Surjectivity is immediate from (4.3).

Hardly surprising, the commuting diagram property (3.6) gives rise to similar algebraic properties of $\widehat{F}_{L, L_{0}}^{(2)}$. They connect $\widehat{F}_{L, L_{0}}^{(2)}$ and several other auxiliary bounded and surjective projectors

$$
\begin{aligned}
& \widehat{\mathrm{G}}_{L, L_{0}}^{(2)}:=\sum_{(l, k) \in \mathcal{S}_{L, L_{0}} \Delta \mathrm{Q}_{l} \otimes \Delta \mathrm{Q}_{k}: L^{2}(D) \otimes L^{2}(D) \rightarrow(\text { curl } \otimes \text { curl }) \widehat{V}_{L, L_{0}},} \Delta \mathrm{F}_{l} \otimes \Delta \mathrm{Q}_{k}: V \otimes L^{2}(D) \rightarrow(\text { Id } \otimes \text { curl }) \widehat{V}_{L, L_{0},} \\
& \widehat{\mathrm{H}}_{L, L_{0}}^{(2)}:=\sum_{(l, k) \in \mathcal{S}_{L, L_{0}}} \Delta \mathrm{Q}_{l} \otimes \Delta \mathrm{F}_{k}: L^{2}(D) \otimes V \rightarrow(\text { curl } \otimes \text { ld }) \widehat{V}_{L, L_{0}}, \\
& \widehat{\mathrm{J}}_{L, L_{0}}^{(2)}:=\sum_{(l, k) \in \mathcal{S}_{L, L_{0}}}
\end{aligned}
$$


where $\Delta Q_{l}:=Q_{l}-Q_{l-1}, l \geq 1, \Delta Q_{0}:=Q_{0}$. Their properties follow by similar arguments as in the proof of Lemma 4.1. Simple computations show that they commute with tensorized versions of curl on $V \otimes V$

$$
\begin{gathered}
(\text { curl } \otimes \text { curl }) \circ \widehat{F}_{L, L_{0}}^{(2)}=\widehat{G}_{L, L_{0}}^{(2)} \circ(\text { curl } \otimes \text { curl }), \\
(\text { Id } \otimes \text { curl }) \circ \widehat{F}_{L, L_{0}}^{(2)}=\widehat{H}_{L, L_{0}}^{(2)} \circ(\text { Id } \otimes \text { curl }), \\
(\text { curl } \otimes \text { Id }) \circ \widehat{F}_{L, L_{0}}^{(2)}=\widehat{J}_{L, L_{0}}^{(2)} \circ(\text { curl } \otimes I d) .
\end{gathered}
$$

\section{Discrete inf-sup conditions}

Our ultimate goal is to show that, asymptotically, the spaces $\widehat{V}_{L, L_{0}}$ offer a uniformly stable Ritz-Galerkin discretization of the tensor product Maxwell operator $\mathrm{A} \otimes \mathrm{A}: V \otimes V \rightarrow V^{\prime} \otimes V^{\prime}$ arising from (2.1) (with associated sesquilinear form $a^{(2)}$ on $V \otimes V)$.

Theorem 5.1 There is a threshold level $L_{0} \in \mathbb{N}$ and $C>0$ such that

$$
\sup _{\widehat{\mathbf{v}}^{(2)} \in \widehat{V}_{L, L_{0}}} \frac{\left|a^{(2)}\left(\widehat{\mathbf{u}}^{(2)}, \widehat{\mathbf{v}}^{(2)}\right)\right|}{\left\|\widehat{\mathbf{v}}^{(2)}\right\|_{V \otimes V}} \geq C\left\|\widehat{\mathbf{u}}^{(2)}\right\|_{V \otimes V} \quad \forall \widehat{\mathbf{u}}^{(2)} \in \widehat{V}_{L, L_{0}}, \forall L \geq L_{0} .
$$

Remark 5.1 The so-called discrete inf-sup condition claimed in Theorem 5.1 directly implies the asymptotic quasi-optimality of sparse tensor Ritz-Galerkin solutions of the second moment equation (1.1) for the Maxwell operator [2]. Thus, a priori estimates can be obtained from best approximation estimates. The latter for sparse tensor finite element spaces are discussed in [15, Sect. 1.4] and they carry over to edge elements.

\subsection{Non-tensor setting}

In order to elucidate the idea behind the proof of Theorem 5.1 let us recall how to establish an asymptotic discrete inf-sup condition for $a(\cdot, \cdot)$ from $(2.1)$ on $V_{L}$, see [10, Sect. 5.2] for a more detailed presentation or [5, Sects. $3 \& 4.1]$ for a more abstract treatment. We start from the generalized Gårding inequality (2.4), which reveals that, given a fixed $\mathbf{u} \in V$,

$$
\mathbf{c}[\mathbf{u}]:=(\Theta+\mathrm{T}) \mathbf{u}, \quad \mathrm{T}:=\mathrm{A}^{-1} \mathrm{~K}: V \rightarrow V,
$$

is a suitable "candidate function" for the continuous inf-sup condition for $a(\cdot, \cdot)$ on $V[10,(5.11)]$.

Now, in the discrete setting we fix $\mathbf{u}_{L} \in V_{L}$, pick

$$
\mathbf{c}_{L}:=\mathrm{F}_{L} \boldsymbol{\Theta} \mathbf{u}_{L}+\mathrm{P}_{L} \mathrm{~T} \mathbf{u}_{L} \in V_{L},
$$


and find

$$
\left\|\mathbf{c}_{L}-\mathbf{c}\left[\mathbf{u}_{L}\right]\right\|_{V} \leq\left\|\left(\mathrm{F}_{L}-\mathrm{Id}\right) \boldsymbol{\Theta} \mathbf{u}_{L}\right\|_{V}+\left\|\left(\mathrm{P}_{L}-\mathrm{Id}\right) \mathrm{T} \mathbf{u}_{L}\right\|_{V} .
$$

Since $\mathrm{T}: V \rightarrow V$ is compact and $\mathrm{P}_{L}-\mathrm{Id} \rightarrow 0$ pointwise for $L \rightarrow \infty$, we can apply [10, Lemma 5.4] to the second term, which yields uniform convergence ${ }^{4}$

$$
\left\|\left(\mathrm{P}_{L}-\mathrm{Id}\right) \circ \mathrm{T}\right\|_{V} \leq v(l)
$$

for a sequence $v: \mathbb{N}_{0} \rightarrow \mathbb{R}^{+}$with $\lim _{L \rightarrow \infty} v(L)=0$.

To deal with the first term in (5.4) observe that, thanks to the commuting diagram property (3.6), $\operatorname{curl}\left(F_{L}-\right.$ Id $) \Theta \mathbf{u}_{L}=\left(Q_{L}-\right.$ Id $) \operatorname{curl} \Theta \mathbf{u}_{L}=\left(Q_{L}-\right.$ Id $) \operatorname{curl} \mathbf{u}_{L}=0$ so that we merely need to estimate its $L^{2}(D)$-norm. Since

$$
\left(\mathrm{F}_{L}-\mathrm{Id}\right) \boldsymbol{\Theta} \mathbf{u}_{L} \stackrel{(2.3)}{=}\left(\mathrm{F}_{L}-\mathrm{Id}\right)\left(2 \mathrm{P}_{X}-\mathrm{Id}\right) \mathbf{u}_{L} \stackrel{(3.7)}{=} 2\left(\mathrm{~F}_{L}-\mathrm{Id}\right) \mathrm{P}_{X} \mathbf{u}_{L},
$$

Lemma 3.1 gives the desired result that, with $C_{F}>0$,

$$
\left\|\left(\mathrm{F}_{L}-\mathrm{Id}\right) \Theta \mathbf{u}_{L}\right\|_{L^{2}(D)} \leq C_{F} h_{L}^{\epsilon}\left\|\mathrm{P}_{X} \mathbf{u}_{L}\right\|_{V} \leq C_{F} h_{L}^{\epsilon}\left\|\mathbf{u}_{L}\right\|_{V} \quad \forall L \in \mathbb{N}_{0},
$$

for some $C_{F}>0$ and with $0<\epsilon \leq 1$ from (3.8).

By (3.5) and the continuity of the other operators involved, we have $\left\|\mathbf{c}_{L}\right\|_{V} \leq$ $C_{c}\left\|\mathbf{u}_{L}\right\|_{V}$ and $\left\|\mathbf{c}_{L}\right\|_{V} \leq C_{c}\left\|\mathbf{c}\left[\mathbf{u}_{L}\right]\right\|_{V}, L \in \mathbb{N}_{0}$, and combining all these estimates we obtain

$$
\begin{aligned}
\sup _{\mathbf{v}_{L} \in V_{L}} \frac{\left|a\left(\mathbf{u}_{L}, \mathbf{v}_{L}\right)\right|}{\left\|\mathbf{v}_{L}\right\|_{V}} & \geq \frac{1}{C_{c}}\left(\frac{\left|a\left(\mathbf{u}_{L}, \mathbf{c}\left[\mathbf{u}_{L}\right]\right)\right|}{\left\|\mathbf{c}\left[\mathbf{u}_{L}\right]\right\|_{V}}-\|\mathrm{A}\|_{V \rightarrow V^{\prime}}\left\|\mathbf{c}_{L}-\mathbf{c}\left[\mathbf{u}_{L}\right]\right\|_{V}\right) \\
& \geq \frac{1}{C_{c}}\left(C_{\text {stab }}-\|\mathrm{A}\|_{V \rightarrow V^{\prime}}\left(v(L)+C_{F} h_{L}^{\epsilon}\right)\right)\left\|\mathbf{u}_{L}\right\|_{V},
\end{aligned}
$$

and, by (3.1), the discrete inf-sup conditions follows when $L$ is sufficiently large.

\subsection{Tensorized setting}

Proof of Theorem 5.1 Let us emulate the policy of Sect. 5.1 for the tensor product operator. As before, initially we fix a "discrete" function $\widehat{\mathbf{u}}^{(2)} \in \widehat{V}_{L, L_{0}}$ in the sparse tensor product trial space. The corresponding "candidate function" that realizes the continuous inf-sup condition for $\mathrm{A} \otimes \mathrm{A}: V \otimes V \rightarrow(V \otimes V)^{\prime}$ is

$$
\mathbf{c}^{(2)}\left[\widehat{\mathbf{u}}^{(2)}\right]=((\boldsymbol{\Theta}+\mathrm{T}) \otimes(\boldsymbol{\Theta}+\mathrm{T})) \widehat{\mathbf{u}}^{(2)} \in V \otimes V,
$$

cf. the proof of [16, Thm. 5.2]. As above, we have to apply suitable projectors to this function, in order to map it into $\widehat{V}_{L, L_{0}}$, and, again as in Sect. 5.1, we may apply

\footnotetext{
${ }^{4}$ For linear operators $V \rightarrow V$ we retain the notation $\|\cdot\|_{V}$ for their norm. The norms of more general linear operators mapping between normed spaces $X \rightarrow Y$ will bear a subscript $X \rightarrow Y:\|\cdot\| X \rightarrow Y$.
} 
different projectors to different terms, and, as above, commuting diagrams for some of the projectors will prove essential. In detail, we start with the splitting

$$
\mathbf{c}^{(2)}\left[\widehat{\mathbf{u}}^{(2)}\right]=(\boldsymbol{\Theta} \otimes \boldsymbol{\Theta}) \widehat{\mathbf{u}}^{(2)}+(\boldsymbol{\Theta} \otimes \mathrm{T}) \widehat{\mathbf{u}}^{(2)}+(\mathrm{T} \otimes \boldsymbol{\Theta}) \widehat{\mathbf{u}}^{(2)}+(\mathrm{T} \otimes \mathrm{T}) \widehat{\mathbf{u}}^{(2)} .
$$

The last three terms can be tackled along the lines of the proof of [16, Thm. 5.2], whereas for the first we have to resort to the particular sparse tensor Fortin projector $\widehat{F}_{L, L_{0}}^{(2)}$ introduced in (4.4); we try the "discrete candidate function"

$$
\begin{aligned}
\widehat{\mathbf{c}}^{(2)}:= & \widehat{\mathrm{F}}_{L, L_{0}}^{(2)}(\boldsymbol{\Theta} \otimes \boldsymbol{\Theta}) \widehat{\mathbf{u}}^{(2)}+\left(\mathrm{F}_{L_{0}} \otimes \mathrm{P}_{L}\right)(\boldsymbol{\Theta} \otimes \mathrm{T}) \widehat{\mathbf{u}}^{(2)} \\
& +\left(\mathrm{P}_{L} \otimes \mathrm{F}_{L_{0}}\right)(\mathrm{T} \otimes \boldsymbol{\Theta}) \widehat{\mathbf{u}}^{(2)}+\left(\mathrm{P}_{L} \otimes \mathrm{P}_{L_{0}}\right)(\mathrm{T} \otimes \mathrm{T}) \widehat{\mathbf{u}}^{(2)} .
\end{aligned}
$$

Now we reap the benefit of the base resolution $L_{0}$ in the definition (4.1) of the sparse tensor edge element space $\widehat{V}_{L, L_{0}}$, because it ensures both

$$
V_{L_{0}} \otimes V_{L} \subset \widehat{V}_{L, L_{0}} \quad \text { and } \quad V_{L} \otimes V_{L_{0}} \subset \widehat{V}_{L, L_{0}},
$$

see Fig. 1, which implies that (5.11) actually defines a function $\widehat{\mathbf{c}}^{(2)} \in \widehat{V}_{L, L_{0}}$. Remember the arguments underlying (5.8); $\widehat{\mathbf{c}}^{(2)} \in \widehat{V}_{L, L_{0}}$ provides a suitable candidate function for the discrete inf-sup condition, if we manage to show

$$
\left\|\widehat{\mathbf{c}}^{(2)}-\mathbf{c}^{(2)}\left[\widehat{\mathbf{u}}^{(2)}\right]\right\|_{V \otimes V} \leq v\left(L_{0}\right)\left\|\widehat{\mathbf{u}}^{(2)}\right\|_{V \otimes V},
$$

with a sequence $v: \mathbb{N}_{0} \rightarrow \mathbb{R}^{+}$that is independent of $\widehat{\mathbf{u}}^{(2)}$ and converges to 0 . This amounts to estimating four different projection errors.

We deal with all terms in (5.11) involving the compact operator $\mathrm{T}$ in the spirit of [16, Sect. 5] and begin by noting that, for instance,

$$
\begin{aligned}
&(I d\left.\otimes I d-F_{L_{0}} \otimes P_{L}\right) \circ(\Theta \otimes T) \\
& \quad=\left(\left(I d-F_{L_{0}}\right) \circ \boldsymbol{\Theta}\right) \otimes T+\left(F_{L_{0}} \circ \boldsymbol{\Theta}\right) \otimes\left(\left(I d-P_{L}\right) \circ T\right) .
\end{aligned}
$$

Therefore, as all operators are bounded in $V$ and the norm of a tensor product of operators is bounded by the product of their norms, we can estimate

$$
\begin{aligned}
& \left\|\left(\mathrm{Id} \otimes \mathrm{Id}-\mathrm{F}_{L_{0}} \otimes \mathrm{P}_{L}\right) \circ(\boldsymbol{\Theta} \otimes \mathrm{T})\right\|_{V \otimes V} \\
& \leq \underbrace{\left\|\left(\mathrm{Id}-\mathrm{F}_{L_{0}}\right) \circ \boldsymbol{\Theta}\right\|_{V}}_{\rightarrow 0 \text { by (5.7) }}\|\mathrm{T}\|_{V}+\left\|\mathrm{F}_{L_{0}}\right\|_{V}\|\boldsymbol{\Theta}\|_{V} \underbrace{\left\|\left(\mathrm{Id}-\mathrm{P}_{L}\right) \circ \mathrm{T}\right\|_{V}}_{\rightarrow 0 \text { by (5.5) }} \rightarrow 0
\end{aligned}
$$

for $L_{0}, L \rightarrow \infty$.

It remains to examine the $V \otimes V$-norm of

$$
\begin{aligned}
& \left((\mathrm{Id} \otimes \mathrm{Id})-\widehat{\mathrm{F}}_{L, L_{0}}^{(2)}\right)(\boldsymbol{\Theta} \otimes \boldsymbol{\Theta}) \widehat{\mathbf{u}}^{(2)} \\
& \stackrel{(2.3)}{=} 4\left((\mathrm{Id} \otimes \mathrm{Id})-\widehat{\mathrm{F}}_{L, L_{0}}^{(2)}\right)\left(\mathrm{P}_{X} \otimes \mathrm{P}_{X}\right) \widehat{\mathbf{u}}^{(2)} \\
& \quad-2\left((\mathrm{Id} \otimes \mathrm{Id})-\widehat{\mathrm{F}}_{L, L_{0}}^{(2)}\right)\left(\mathrm{Id} \otimes \mathrm{P}_{X}\right) \widehat{\mathbf{u}}^{(2)}
\end{aligned}
$$




$$
\begin{aligned}
& -2\left((I d \otimes I d)-\widehat{F}_{L, L_{0}}^{(2)}\right)\left(P_{X} \otimes I d\right) \widehat{\mathbf{u}}^{(2)} \\
& +\underbrace{\left((I d \otimes I d)-\widehat{F}_{L, L_{0}}^{(2)}\right)(I d \otimes I d) \widehat{\mathbf{u}}^{(2)}}_{=0 !},
\end{aligned}
$$

where we used $\boldsymbol{\Theta}=2 \mathrm{P}_{X}-$ Id from (2.3). Pay attention that the last term vanishes due to the projector property of $\widehat{F}_{L, L_{0}}^{(2)}$.

Estimating the $V \otimes V$-norm of the other terms turns out to be challenging. To begin with, remember that this norm comprises four parts

$$
\begin{aligned}
\left\|\mathbf{v}^{(2)}\right\|_{V \otimes V}^{2}= & \|(\text { curl } \otimes \mathbf{c u r l}) \mathbf{v}^{(2)} \|_{L^{2}(D) \otimes L^{2}(D)}^{2} \\
& +\|(\text { curl } \otimes \mathbf{l d}) \mathbf{v}^{(2)} \|_{L^{2}(D) \otimes L^{2}(D)}^{2} \\
& +\|(\text { ld } \otimes \mathbf{c u r l}) \mathbf{v}^{(2)} \|_{L^{2}(D) \otimes L^{2}(D)}^{2} \\
& +\|(\text { ld } \otimes \mathbf{l d}) \mathbf{v}^{(2)} \|_{L^{2}(D) \otimes L^{2}(D)}^{2} .
\end{aligned}
$$

Inevitably, we have to examine the various combinations of terms in (5.14a)-(5.14d) and contributions to the norm in (5.15a)-(5.15d). Inherent symmetries make several of them amenable to the same arguments and we are going to skip parallel developments.

(1) (5.14a) \& (5.15d): With convergence of the infinite sum understood pointwise in $V \otimes V$, we have the error representation

$$
(\mathrm{Id} \otimes \mathrm{Id})-\widehat{\mathrm{F}}_{L, L_{0}}^{(2)}=\sum_{(l, k) \notin \mathcal{S}_{L, L_{0}}} \Delta \mathrm{F}_{l} \otimes \Delta \mathrm{F}_{k},
$$

which we conclude from the direct sum decomposition of $V: \mathbf{u}=\sum_{l=0}^{\infty} \Delta \mathrm{F}_{l} \mathbf{u}, \mathbf{u} \in V$, along with the pointwise convergence $\mathrm{F}_{l} \rightarrow$ Id for $l \rightarrow \infty$ [10, Lemma 5.5].

$$
\begin{aligned}
\left\|\sum_{(l, k) \notin \mathcal{S}_{L, L_{0}}}\left(\Delta \mathrm{F}_{l} \otimes \Delta \mathrm{F}_{k}\right)\left(\mathrm{P}_{X} \otimes \mathrm{P}_{X}\right) \widehat{\mathbf{u}}^{(2)}\right\|_{L^{2}(D) \otimes L^{2}(D)} \\
=\left\|\sum_{(l, k) \notin \mathcal{S}_{L, L_{0}}}\left(\left(\Delta \mathrm{F}_{l} \circ \mathrm{P}_{X}\right) \otimes\left(\Delta \mathrm{F}_{k} \circ \mathrm{P}_{X}\right)\right) \widehat{\mathbf{u}}^{(2)}\right\|_{L^{2}(D) \otimes L^{2}(D)} \\
\sum_{\leq}^{\operatorname{Lemma} 3.1} C h_{(l, k) \notin \mathcal{S}_{L, L_{0}}} h_{k}^{\epsilon}\left\|\widehat{\mathbf{u}}^{(2)}\right\|_{V \otimes V} \stackrel{(3.1)}{\leq} C h_{0}^{2 \epsilon}\left\|\widehat{\mathbf{u}}^{(2)}\right\|_{V \otimes V} \underbrace{\sum_{(l, k) \notin \mathcal{S}_{L, L_{0}}} q^{\epsilon(l+k)}}_{\rightarrow 0 \text { for } L \rightarrow \infty} .
\end{aligned}
$$

2 (5.14a) \& (5.15b): The commuting diagrams underlying (4.8) is key to handling this contribution, because they pave the way for reformulating

$$
(\operatorname{curl} \otimes I d) \circ\left(I d \otimes I d-\widehat{F}_{L, L_{0}}^{(2)}\right) \circ\left(P_{X} \otimes P_{X}\right) \widehat{\mathbf{u}}^{(2)}
$$




$$
\begin{aligned}
& \stackrel{(\mathrm{A})}{=} \sum_{\substack{(l, k) \notin \mathcal{S}_{L, L_{0}} \\
l \leq L}}\left(\left(\Delta \mathrm{Q}_{l} \circ \text { curl }\right) \otimes\left(\Delta \mathrm{F}_{k} \circ \mathrm{P}_{X}\right)\right) \widehat{\mathbf{u}}^{(2)} \\
& \stackrel{(\mathrm{B})}{=} \sum_{l=0}^{L} \sum_{k=1+L_{0}+L-l}^{\infty}\left(\left(\Delta \mathrm{Q}_{l} \circ \text { curl }\right) \otimes\left(\Delta \mathrm{F}_{k} \circ \mathrm{P}_{X}\right)\right) \widehat{\mathbf{u}}^{(2)} \\
& \stackrel{(\mathrm{C})}{=} \sum_{l=0}^{L}\left(\left(\Delta \mathrm{Q}_{l} \circ \text { curl }\right) \otimes\left(\left(\mathrm{ld}-\mathrm{F}_{L_{0}+L-l}\right) \circ \mathrm{P}_{X}\right)\right) \widehat{\mathbf{u}}^{(2)}
\end{aligned}
$$

The identity (A) arises from using (4.8), curl $\circ \mathrm{P}_{X}=$ curl, together with an error representation analogous to (5.16). Moreover, the extra restriction $l \leq L$ on the index range results from the trivial fact that $\left(\operatorname{curl} \otimes \mathrm{P}_{X}\right) \widehat{\mathbf{u}}^{(2)} \in \mathcal{W}_{h}^{2,0}\left(\mathcal{T}_{L}\right) \otimes V$ and $\Delta \mathrm{Q}_{l}\left(\mathcal{W}_{h}^{2,0}\left(\mathcal{T}_{L}\right)\right)=0$, whenever $l>L$. Identity $(\mathrm{B})$ is a consequence of the definition of $\mathcal{S}_{L, L_{0}}$, and (C) reflects a telescopic sum. Invoking (5.7) we obtain

$$
\begin{aligned}
& \left\|(\mathbf{c u r l} \otimes \mathrm{Id}) \circ\left(\mathrm{Id} \otimes \mathrm{Id}-\widehat{\mathrm{F}}_{L, L_{0}}^{(2)}\right) \circ\left(\mathrm{P}_{X} \otimes \mathrm{P}_{X}\right) \widehat{\mathbf{u}}^{(2)}\right\|_{L^{2}(D) \otimes L^{2}(D)} \\
& \leq \sum_{l=0}^{L} \underbrace{\left\|\Delta \mathrm{Q}_{l} \circ \mathbf{c u r l}\right\|_{V \rightarrow L^{2}(D)}}_{\leq 1} \underbrace{\left\|\left(\mathrm{Id}-\mathrm{F}_{L_{0}+L-l}\right) \circ \mathrm{P}_{X}\right\|_{V \rightarrow L^{2}(D)}}_{\text {apply }(5.7)}\left\|\widehat{\mathbf{u}}^{(2)}\right\|_{V \otimes V} \\
& \leq C\left\|\widehat{\mathbf{u}}^{(2)}\right\|_{V \otimes V} \underbrace{\left(\sum_{l=L_{0}}^{L} h_{l}^{\epsilon}\right)}_{\rightarrow 0 \text { for } L_{0} \rightarrow \infty} .
\end{aligned}
$$

The next term (5.14a) \& (5.15c) has a similar structure and can be treated alike.

(3) The final term (5.14a) \& (5.15a) is killed by the commuting diagram property (4.8):

$$
\begin{aligned}
& (\text { curl } \otimes \text { curl })\left(I d \otimes I d-\widehat{F}_{L, L_{0}}^{(2)}\right)\left(P_{X} \otimes P_{X}\right) \widehat{\mathbf{u}}^{(2)} \\
& =\left(I d \otimes I d-\widehat{G}_{L, L_{0}}^{(2)}\right)(\text { curl } \otimes \operatorname{curl})\left(P_{X} \otimes P_{X}\right) \widehat{\mathbf{u}}^{(2)} \\
& =\left(I d \otimes I d-\widehat{G}_{L, L_{0}}^{(2)}\right)(\operatorname{curl} \otimes \operatorname{curl}) \widehat{\mathbf{u}}^{(2)}=0,
\end{aligned}
$$

thanks to the projector property of $\widehat{G}_{L, L_{0}}^{(2)}$, see (4.5).

4 (5.14c) \& (5.15d): We start from the error representation (5.16) and, as in Step (2), continue with the identity

$$
\begin{aligned}
\left(\mathrm{Id} \otimes \mathrm{Id}-\widehat{\mathrm{F}}_{L, L_{0}}^{(2)}\right)\left(\mathrm{P}_{X} \otimes \mathrm{Id}\right) \widehat{\mathbf{u}}^{(2)} & =\sum_{(l, k) \notin \mathcal{S}_{L, L_{0}}}\left(\Delta \mathrm{F}_{l} \otimes \Delta \mathrm{F}_{k}\right)\left(\mathrm{P}_{X} \otimes \mathrm{Id}\right) \widehat{\mathbf{u}}^{(2)} \\
& =\sum_{\substack{(l, k) \notin \mathcal{S}_{L, L_{0}} \\
k \leq L}}\left(\left(\Delta \mathrm{F}_{l} \circ \mathrm{P}_{X}\right) \otimes \Delta \mathrm{F}_{k}\right) \widehat{\mathbf{u}}^{(2)}
\end{aligned}
$$




$$
\begin{aligned}
& =\sum_{k=0}^{L} \sum_{l=1+L_{0}+k-L}^{\infty}\left(\left(\Delta \mathrm{F}_{l} \circ \mathrm{P}_{X}\right) \otimes \Delta \mathrm{F}_{k}\right) \widehat{\mathbf{u}}^{(2)} \\
& =\sum_{k=0}^{L}\left(\left(\left(\mathrm{ld}-\mathrm{F}_{L_{0}+k-L}\right) \circ \mathrm{P}_{X}\right) \otimes \Delta \mathrm{F}_{k}\right) \widehat{\mathbf{u}}^{(2)}
\end{aligned}
$$

Combined with (5.7) it yields the bound

$$
\begin{aligned}
& \left\|\left(\mathrm{Id} \otimes \mathrm{Id}-\widehat{\mathrm{F}}_{L, L_{0}}^{(2)}\right)\left(\mathrm{P}_{X} \otimes \mathrm{Id}\right) \widehat{\mathbf{u}}^{(2)}\right\|_{L^{2}(D) \otimes L^{2}(D)} \\
& \leq \sum_{k=0}^{L} \underbrace{\left\|\left(\mathrm{Id}-\mathrm{F}_{L_{0}+L-k}\right) \circ \mathrm{P}_{X}\right\|_{V \rightarrow L^{2}(D)}}_{\text {apply }(5.7)} \underbrace{\left\|\Delta \mathrm{F}_{k}\right\|_{V}}_{\text {bounded }}\left\|\widehat{\mathbf{u}}^{(2)}\right\|_{V \otimes V} \\
& \leq C\left\|\widehat{\mathbf{u}}^{(2)}\right\|_{V \otimes V} \underbrace{\sum_{k=L_{0}}^{L_{0}+L} h_{k}^{\epsilon}}_{\rightarrow 0 \text { for } L_{0} \rightarrow \infty} .
\end{aligned}
$$

(5 (5.14c) \& (5.15c): Here we use the commuting diagram behind (4.9) and, as in Step (2), get

$$
\begin{aligned}
(\mathrm{Id} & \otimes \mathbf{c u r l})\left(\mathrm{Id} \otimes \mathrm{ld}-\widehat{\mathrm{F}}_{L, L_{0}}^{(2)}\right)\left(\mathrm{P}_{X} \otimes \mathrm{Id}\right) \widehat{\mathbf{u}}^{(2)} \\
& =\sum_{\substack{(l, k) \notin \mathcal{S}_{L, L_{0}} \\
k \leq L}}\left(\left(\Delta \mathrm{F}_{l} \circ \mathrm{P}_{X}\right) \otimes\left(\Delta \mathrm{Q}_{k} \circ \mathbf{c u r l}\right)\right) \widehat{\mathbf{u}}^{(2)} \\
& =\sum_{k=0}^{L} \sum_{l=1+L_{0}+L-k}^{\infty}\left(\left(\Delta \mathrm{F}_{l} \circ \mathrm{P}_{X}\right) \otimes\left(\Delta \mathrm{Q}_{k} \circ \text { curl }\right)\right) \widehat{\mathbf{u}}^{(2)} \\
& =\sum_{k=0}^{L}\left(\left(\left(\mathrm{Id}-\mathrm{F}_{L_{0}+L-k}\right) \circ \mathrm{P}_{X}\right) \otimes\left(\Delta \mathrm{Q}_{k} \circ \text { curl }\right)\right) \widehat{\mathbf{u}}^{(2)}
\end{aligned}
$$

As before, this permits us to continue

$$
\begin{aligned}
& \left\|(\mathbf{I d} \otimes \mathbf{c u r l})\left(\mathrm{Id} \otimes \mathrm{Id}-\widehat{\mathrm{F}}_{L, L_{0}}^{(2)}\right)\left(\mathrm{P}_{X} \otimes \mathrm{Id}\right) \widehat{\mathbf{u}}^{(2)}\right\|_{L^{2}(D) \otimes L^{2}(D)} \\
& \leq \sum_{k=0}^{L} \underbrace{\left\|\left(\mathrm{Id}-\mathrm{F}_{L_{0}+L-k}\right) \circ \mathrm{P}_{X}\right\|_{V \rightarrow L^{2}(D)}}_{\operatorname{apply}(5.7)} \underbrace{\left\|\Delta \mathrm{Q}_{k} \circ \mathbf{c u r l}\right\|_{V \rightarrow L^{2}(D)}}_{\leq 1}\left\|\widehat{\mathbf{u}}^{(2)}\right\|_{V \otimes V} \\
& \leq C\left\|\widehat{\mathbf{u}}^{(2)}\right\|_{V \otimes V} \underbrace{\sum_{k=L_{0}}^{L_{0}+L} h_{k}^{\epsilon}}_{\rightarrow 0 \text { for } L_{0} \rightarrow \infty} .
\end{aligned}
$$


6 (5.14c) \& (5.15b): We recall the commuting diagram (4.8), which delivers

$$
\begin{aligned}
& (\text { curl } \otimes I d)\left(I d \otimes I d-\widehat{F}_{L, L_{0}}^{(2)}\right)\left(P_{X} \otimes I d\right) \widehat{\mathbf{u}}^{(2)} \\
& =\left(I d \otimes I d-\widehat{J}_{L, L_{0}}^{(2)}\right)(\text { curl } \otimes I d)\left(P_{X} \otimes I d\right) \widehat{\mathbf{u}}^{(2)} \\
& =\left(I d \otimes I d-\widehat{J}_{L, L_{0}}^{(2)}\right)(\text { curl } \otimes I d) \widehat{\mathbf{u}}^{(2)}=0,
\end{aligned}
$$

due to the projector property of $\widehat{J}_{L, L_{0}}^{(2)}$, see (4.7).

7 (5.14c) \& (5.15a): Here we rely on (4.8) and get

$$
\begin{aligned}
& (\text { curl } \otimes \text { curl })\left(I d \otimes I d-\widehat{F}_{L, L_{0}}^{(2)}\right)\left(P_{X} \otimes I d\right) \widehat{\mathbf{u}}^{(2)} \\
& =\left(I d \otimes I d-\widehat{G}_{L, L_{0}}^{(2)}\right)(\text { curl } \otimes \text { curl })\left(P_{X} \otimes I d\right) \widehat{\mathbf{u}}^{(2)} \\
& =\left(I d \otimes I d-\widehat{G}_{L, L_{0}}^{(2)}\right)(\text { curl } \otimes \text { curl }) \widehat{\mathbf{u}}^{(2)}=0,
\end{aligned}
$$

where we used that $\widehat{G}_{L, L_{0}}^{(2)}$ is a surjective projector onto (curl $\otimes$ curl) $\widehat{V}_{L, L_{0}}$.

\section{Extensions}

We deliberately restricted ourselves to a simple setting in order to keep technical complexity at bay. Nevertheless the developments in this article convey all the main ideas needed to tackle other situations:

\subsection{Higher order moments}

It is straightforward but tedious to extend the estimates to the case of $k$-fold tensor product operators

$$
\mathrm{A}^{(k)}=\underbrace{\mathrm{A} \otimes \ldots \otimes \mathrm{A}}_{k \text { times }}, \quad k>2 .
$$

For the Helmholtz operator this case is treated in [15, Sect. 1] and for mixed problems we refer to [4].

\subsection{Electric field integral equation (EFIE)}

In this case we work in the trace space $V:=H^{-\frac{1}{2}}\left(\operatorname{div}_{\Gamma}, \Gamma\right)$ [7, Sect. 2] on a closed orientable polyhedral surface $\Gamma$ and deal with the non-positive sesquilinear form, see [7, Sect. 7], and [6],

$$
\begin{aligned}
a(\boldsymbol{\xi}, \boldsymbol{\eta})= & \int_{\Gamma} \int_{\Gamma} \frac{\exp (-i k|\boldsymbol{x}-\boldsymbol{y}|)}{4 \pi|\boldsymbol{x}-\boldsymbol{y}|}(\boldsymbol{\xi}(\boldsymbol{x}) \overline{\boldsymbol{\eta}}(\boldsymbol{y}) \\
& \left.-k^{-2} \operatorname{div}_{\Gamma} \boldsymbol{\xi}(\boldsymbol{x}) \operatorname{div}_{\Gamma} \overline{\boldsymbol{\eta}}(\boldsymbol{y})\right) \mathrm{d} S(\boldsymbol{y}) \mathrm{d} S(\boldsymbol{x}), \quad \boldsymbol{\xi}, \boldsymbol{\eta} \in V,
\end{aligned}
$$


which is discretized using surface edge elements (also known as Raviart-Thomas boundary elements or RWG elements) [7, Sect. 8].

Parallel to the considerations of Sect. 3 we can consider $L^{2}(\Gamma)$-orthogonal discrete Hodge decompositions [11, Sect. 6] and use them to define 2D analogues of the Fortin projectors $F_{l}$. A key observation from [11, Lemma 2.3] is that the range of the $H^{-\frac{1}{2}}\left(\operatorname{div}_{\Gamma}, \Gamma\right)$-counterpart of the projection $\mathrm{P}_{X}$ will be compactly embedded in the space $L_{\mathbf{t}}^{2}(\Gamma)$ of square-integrable tangential vector fields on $\Gamma$. In addition [11, Lemma 6.2] provides an approximation result that can replace Lemma 3.1; basically, [11] is about adapting the developments of Sects. 3 and 5.1 to the EFIE. Appealing to these results, all estimates from Sect. 5 essentially remain valid and no new ideas are required. Thus, asymptotic quasi-optimality of sparse tensor RitzGalerkin approximation of the tensorized EFIE-operator can be regarded as settled.

\subsection{Curvilinear polyhedra and higher order edge elements}

Also in this case "nil novi sub sole": Mapping techniques will take care of nonpolyhedral domains. Higher order edge elements [10, Sect. 3.4] allow for exactly the same techniques as discussed for the lowest order case. Beware that all constants will depend on the polynomial degree, however.

\section{References}

1. Arnold, D., Falk, R., Winther, R.: Finite element exterior calculus: from Hodge theory to numerical stability. Bull. Am. Math. Soc. 47, 281-354 (2010)

2. Babuška, I.: Error bounds for the finite element method. Numer. Math. 16, 322-333 (1971)

3. Boffi, D.: Fortin operator and discrete compactness for edge elements. Numer. Math. 87, 229-246 (2000)

4. Bonizzoni, F., Nobile, F., Buffa, A.: Moment equations for the mixed formulation of the Hodge Laplacian with stochastic data. Technical Report 31.2012 \& Erratum, EPFL-ARTICLE181594, MATHICSE, EPFL Lausanne, August 2012. Erratum published in November 2012 http://mathicse.epfl.ch/page-68906-en.html

5. Buffa, A.: Remarks on the discretization of some non-positive operators with application to heterogeneous Maxwell problems. SIAM J. Numer. Anal. 43, 1-18 (2005)

6. Buffa, A., Christiansen, S.: The electric field integral equation on Lipschitz screens: Definition and numerical approximation. Numer. Math. 94, 229-267 (2002)

7. Buffa, A., Hiptmair, R.: Galerkin boundary element methods for electromagnetic scattering. In: Ainsworth, M., Davis, P., Duncan, D., Martin, P., Rynne, B. (eds.) Topics in Computational Wave Propagation. Direct and Inverse Problems. Lecture Notes in Computational Science and Engineering, vol. 31, pp. 83-124. Springer, Berlin (2003)

8. Christiansen, S.: Résolution des équations intégrales pour la diffraction d'ondes acoustiques et électromagnétiques: Stabilisation d'algorithmes itératifs et aspects de ánalyse numérique. $\mathrm{PhD}$ thesis, Ecole Polytechnique, Paris, France (2002)

9. Christiansen, S.H., Winther, R.: Smoothed projections in finite element exterior calculus. Math. Comput. 77, 813-829 (2008)

10. Hiptmair, R.: Finite elements in computational electromagnetism. Acta Numer. 11, 237-339 (2002)

11. Hiptmair, R., Schwab, C.: Natural boundary element methods for the electric field integral equation on polyhedra. SIAM J. Numer. Anal. 40, 66-86 (2002)

12. Nédélec, J.: Mixed finite elements in $\mathbb{R}^{3}$. Numer. Math. 35, 315-341 (1980) 
13. Schatz, A.: An observation concerning Ritz-Galerkin methods with indefinite bilinear forms. Math. Comput. 28, 959-962 (1974)

14. Schöberl, J.: A posteriori error estimates for Maxwell equations. Math. Comput. 77, 633-649 (2008)

15. Schwab, C., Gittelson, C.J.: Sparse tensor discretizations of high-dimensional parametric and stochastic PDEs. Acta Numer. 20, 291-467 (2011)

16. von Petersdorff, T., Schwab, C.: Sparse finite element methods for operator equations with stochastic data. Appl. Math. 51, 145-180 (2006) 